\title{
SMOKE DETECTION IN VIDEO BASED ON MOTION AND CONTRAST
}

\author{
N. BROVKO ${ }^{1}$, R. BOGUSH ${ }^{1}$, S. ABLAMEYKO ${ }^{2}$ \\ ${ }^{1}$ Polotsk State University, 29, Blokhin str., Novopolotsk, Belarus \\ ${ }^{2}$ Belarusian State University, 4, Nezavisimosti av., Minsk, Belarus
}

Tóm tắt. Bài báo đề xuất một thuật toán hữu hiệu phát hiện khói trong video màu từ máy quay camera tĩnh. Thuật toán xem xét các đặc trưng động và tĩnh của khói bao gồm các bước cơ bản: Tiền sử lí; Các miền di chuyển chậm và các phân đoạn ảnh điểm trong khung dữ liệu nhập dựa trên khấu trừ thích nghi; Hợp nhất các miền dịch chuyển chậm với các điểm ảnh thành các giọt nước; Phân loại các giọt nước. Bài báo đã sử dụng phương pháp khấu trừ thích nghi trên từng giai đoạn phát triển khói. Phân loại các giọt nước di động dựa trên tính toán các dòng quang học, trên sự phân tích tương phản Weber và có tính đến hướng khói lan tỏa. Phối hợp giám sát các hình ảnh video thật được sử dụng để phát hiện khói. Các kết quả thực nghiệm cũng được đưa ra.

\begin{abstract}
An efficient smoke detection algorithm on color video sequences obtained from a stationary camera is proposed. Our algorithm considers dynamic and static features of smoke and composed of basic steps: preprocessing; slowly moving areas and pixels segmentation in a current input frame based on adaptive background subtraction; merge slowly moving areas with pixels into blobs; classification of the blobs obtained before. We use adaptive background subtraction at a stage of moving detection. Moving blobs classification is based on optical flow calculation, Weber contrast analysis and takes into account primary direction of smoke propagation. Real video surveillance sequences are used for smoke detection with utilization our algorithm. A set of experimental results are presented in the paper.
\end{abstract}

Keywords. smoke detection, video sequences, background subtraction, Weber contrast analysis

\section{INTRODUCTION}

Reliable and early fire detection on open spaces, in buildings, in territories of the industrial enterprises are an important feature to make any system of fire safety. Traditional fire detectors which have been widely applied in the buildings are based on infrared sensors, optical sensors, or ion sensors that depend on certain characteristics of fire, such as smoke, heat, or radiation. Such detection approaches require a position of sensor in very close proximity to fire or smoke and often give out false alarms. Thus they may be not reliable and cannot be applied into open spaces and larger areas.

Effective systems for early fire detection into open spaces are using technologies such as image and video processing [1, 2], radio-acoustic sounding (RASS) [3], light detection and ranging (LIDAR) [4]. Due to the rapid developments in digital camera technology and video processing techniques currently intelligent video surveillance systems are installed in various public places for monitoring. Therefore there is a noticeable trend to use such systems for 
early fire detection with special software applied [5]. Smoke detection is rather for fire alarm systems when large and open areas are monitored, because the source of the fire and flames cannot always be captured. Whereas smoke of an uncontrolled fire can be easily observed by a camera even if the flames are not visible. This results in early detection of fire before it spreads around.

Motion and color are two usually used important features for detecting smoke on the video sequences. Motion information provides a key as the precondition to locate the possible smoke regions. The algorithm of background subtraction is traditionally applied to movement definition in video sequence $[6,8]$. Common technique is using adaptive Gaussian Mixture Model to approximate the background modeling process [6, 7].

The existing algorithms of smoke detection combine various smoky properties based on classifiers. In the paper [6], the energy ratio and the color blending have been combined using a Bayesian classifier to detect smoke on the scene. The algorithm in paper [5] is mainly based on determining the edge regions whose wavelet sub band energies decrease with time and wavelet based contour analysis of possible flame regions. These regions are then analyzed along with their corresponding background regions with respect to their RGB and chrominance values.

In [9], optical flow calculation is applied to detection of movement of a smoke. Lacks of the present approach are high sensitivity to noise and low performance. Algorithms based on color and dynamic characteristics of a smoke are applied to classify the given moving blobs. In [10] the algorithm comparative evaluation of the histogram-based pixel level classification is considered. Based on this algorithm the training set of video sequences on which there is a smoke is applied to the analysis. In [11] the algorithm uses estimated motion orientation with accumulation intensity for disturbance of artificial lights and non-smoke moving objects elimination.

Color information is also used to identify smoke in video. Smoke color changes at the different stages of ignition and depending on a burning material is distributed in a range from almost transparent white to saturated gray and black. In [6] decrease in value of chromatic components $\mathrm{U}$ and $\mathrm{V}$ of color space YUV is estimated.

Image regions containing smoke are characterized with a dynamic texture (changing texture of an image over time) [12]. In [13] a model of the instantaneous motion maps allows to track motion textures using the conditional Kullback-Leibler divergence between mixedstate probability densities, which allows to estimate the position using a statistical matching approach.

In this paper, we propose an algorithm for smoke detection on color video sequences obtained from a stationary camera. Our algorithm consists of the following steps: preprocessing; slowly moving areas and pixels segmentation in a current input frame based on adaptive background subtraction; merge slowly moving areas with pixels into blobs; classification of the blobs obtained before. We use adaptive background subtraction at a stage of moving detection. Moving blobs classification is based on optical flow calculation, Weber contrast analysis and takes into account primary direction of smoke propagation.

\section{ALGORITHM DESCRIPTION}

The proposed algorithm uses motion and contrast as the two key features for smoke detection. Motion is a primary sign and used at the beginning for extraction from a current frame 
of candidate areas. In addition we consider a direction of smoke distribution the movement estimation based on the optical flow is applied. The relation of smoke intensity to background intensity above than at objects with similar behavior, such as a fog, shadows from slowly moving objects and patches of light. Therefore, contrast calculated with Weber formula is a good distinctive sign for a smoke. The algorithm is a group of the following modules as showed in Figure 2.1

Consecutive frames $I_{t-2}, I_{t-1}, I_{t}$ and $I_{t-1}$ obtained from the stationary video surveillance camera are entered to an input of the preprocessing block. This block carries out some transformations, which improve contrast qualities of the input frames and reduce calculations. Then adaptive background subtraction is applied to extract from the frame $I_{t-1}$ of slowly moving areas and pixels of the so-called foreground. The background subtraction adaptive algorithm considers that a smoke gradually is mixed to a background. Then the connected components analysis is used to clear the foreground noise and to merge the slowly moving areas with pixels into blobs. The received connected blobs are transferred into the classification block for Weber contrast analysis. At the same times the connected blobs are entered to an input of the block for optical flow calculation. Finally, the classification block processes the information to obtain the final result of smoke detection.

\subsection{Frame preprocessing}

The preprocessing block applies some methods of image processing, which increase the performance of the proposed detection algorithm and reduce false alarms. Frame preprocessing block comprises three steps: grayscale transformation, histogram equalization and the discrete wavelet of the current input frame. Cameras and image sensors must usually deal not only with the contrast on a scene, but also with the image sensors exposure to the resulting light on that scene. Histogram equalization is a most commonly used method for improving contrast image characteristics. To resize the image and to remove high frequencies on horizontal, vertical and diagonal details the discrete wavelet transform to Haar basis is applied. Wavelet transform to Haar basis is the simplest and the fastest [14] algorithm that is important for systems of video processing. Figure 2.2 shows the results for this step of algorithm.

\subsection{Slowly moving areas and pixels segmentation}

In the course of the distribution a smoke is being gradually blended to the background. Our adaptive algorithm of background subtraction considers this characteristic of a smoke and is based on the ideas of $[7,15]$. A background image $B_{t}$ at time instant $t$ is recursively estimated from the image frame $I_{t-1}$ and the background image $B_{t-1}$ of the video as follows [15]:

$$
B_{t}(x, y)=\left\{\begin{array}{l}
\alpha B_{t-1}(x, y)+(1-\alpha) T_{t-1}(x), \text { if }(x, y) \text { is moving } \\
B_{t-1}(x, y), \text { if }(x, y) \text { is stationary }
\end{array}\right.
$$

where $(x, y)$ represent a pixel video frame and $\alpha$ is a adaptation parameter between 0 and 1 . As the area of a smoke frame by frame grows slowly that the pixels belonging to a smoke, quickly did not fix in a background, value $\alpha$ should close to 1 .

At the initial moment of time $B_{0}(x, y)=I_{0}(x, y)$. Pixel $(x, y)$ belongs to moving object if the following condition is satisfied [15]: 


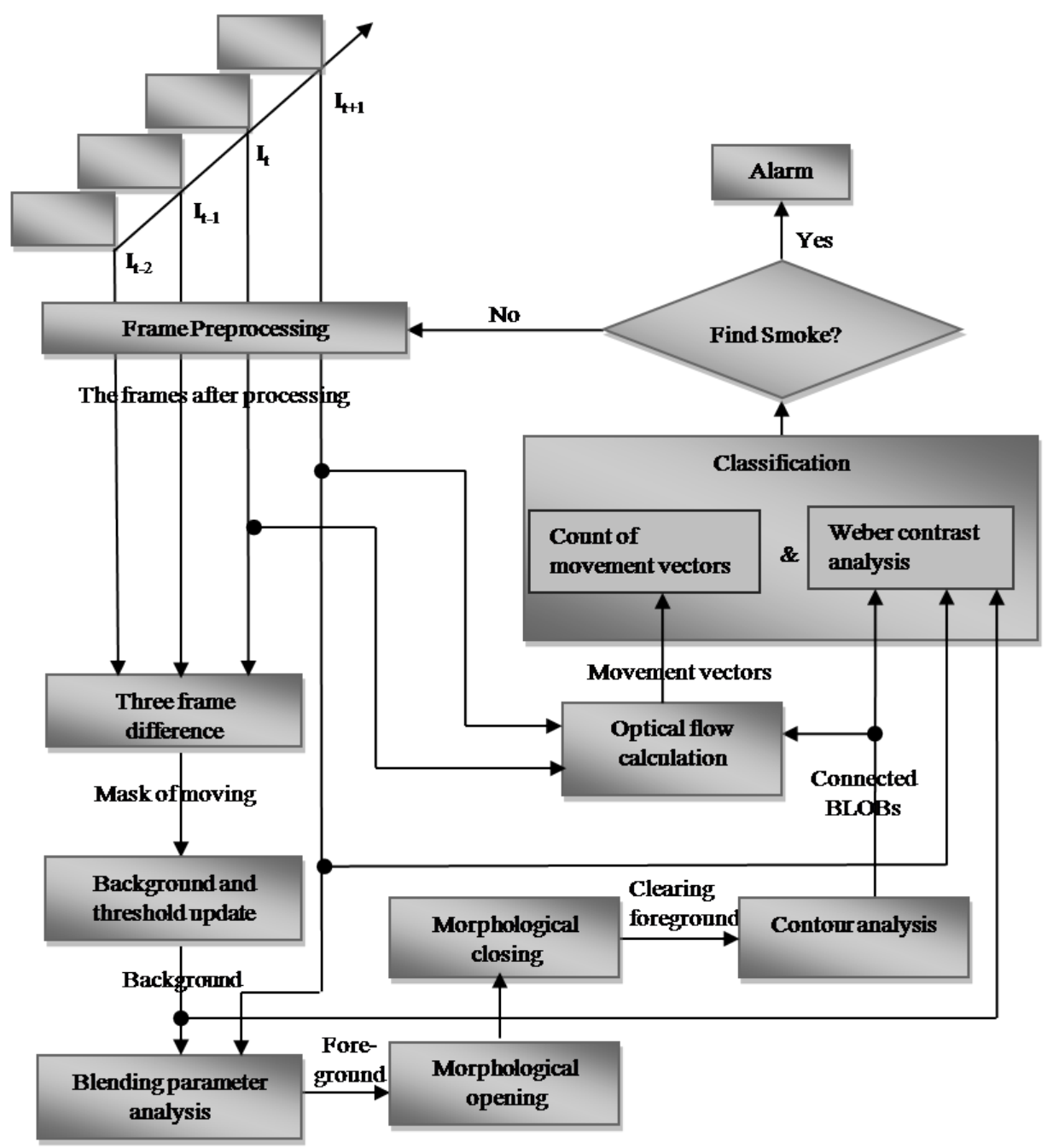

Figure 2.1. Flow chart of our proposed algorithm

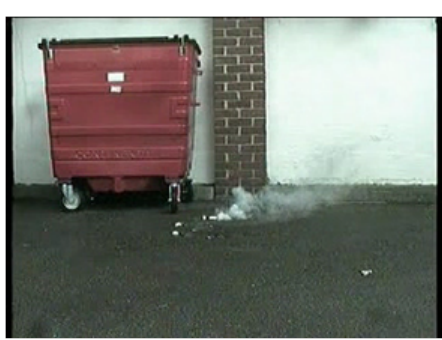

(a)

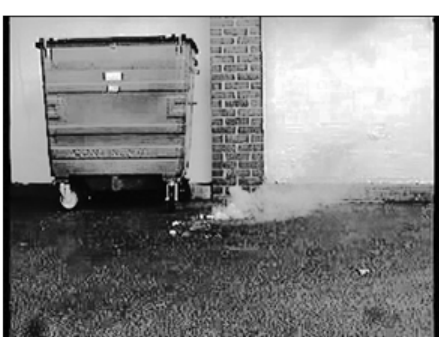

(b)

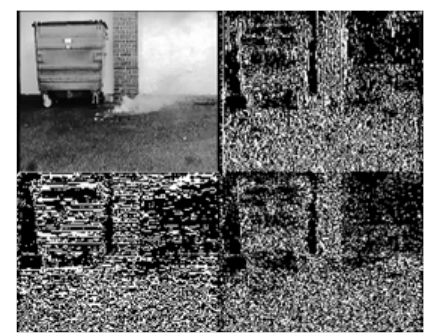

(c)

Figure 2.2. The current frame (a), histogram equalization (b) and the Haar transform of the current frame (c) 


$$
\left(\left|I_{t}(x, y)-I_{t-1}(x, y)\right|>T_{t-1}(x, y)\right) \&\left(\left|I_{t}(x, y)-I_{t-2}(x, y)\right|>T_{t-1}(x, y)\right),
$$

where $I_{t-2}(x, y), I_{t-1}(x, y), I_{t}(x, y)$ values of intensity of pixel $(x, y)$ at time instant $t-2, t-1$ and $t$ respectively;

$T_{t}(x, y)$ is adaptive threshold for pixel $(x, y)$ at time instant $t$ calculated as follows:

$$
T_{t}(x, y)=\left\{\begin{array}{l}
\alpha T_{t-1}(x, y)+(1-\alpha)\left(5 \times\left|I_{t-1}(x, y)-B_{t-1}(x, y)\right|\right), \text { if }(x, y) \text { is moving } \\
T_{t-1}(x, y), \text { if }(x, y) \text { is stationary. }
\end{array}\right.
$$

At the initial moment of time $T_{0}(x, y)=$ const $>0$.

Accurate separating of a foreground object from the background is the main task of digital matting. Porter and Duff [17] introduced the blending parameter (so-called alpha channel) as a solution of this problem and a mean to control the linear combination of foreground and background components. Mathematically the current frame $I_{t+1}$ is modeled as a combination of foreground $F_{t+1}$ and background $B_{t}$ components using the blending parameter $\beta$ :

$$
I_{t+1}(x, y)=\beta F_{t+1}(x, y)+(1-\beta) b_{t}(x, y) .
$$

For opaque objects value of $\beta$ is equal to 1 , for transparent objects value of $\beta$ is equal to 0 and for the semitransparent objects, such as smoke, value of $\beta$ lays in a range from 0 to 1 . As it is shown further in this section we have experimentally established the optimum value for $\beta$, to be equal to 0.38 .

So, as soon as we have obtained $B_{t}$ component on background update step, current frame $I_{t+1}$ and set $\beta$ to 0.38 , we can estimate the foreground component $F_{t+1}$. Then we apply the threshold processing to receive the binary foreground $F_{b i n}$ :

$$
F_{b i n}=\left\{\begin{array}{l}
1, \text { if }\left(F_{t+1}>245\right) \\
0, \text { otherwise }
\end{array}\right.
$$

The figure 2.3 shows the results of adaptive background subtraction and threshold of foreground component $F_{t+1}$.

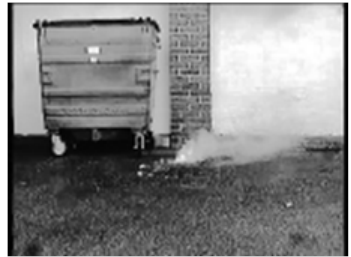

(a)

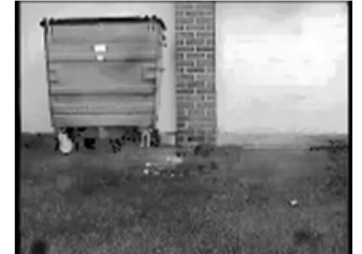

(b)

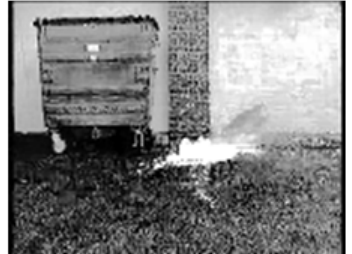

(c)

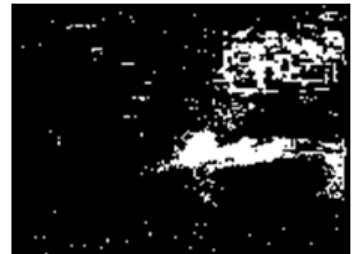

(d)

Figure 2.3. The current frame $I_{t+1}$ (a), the background component $B_{t}(\mathrm{~b})$, the foreground component $F_{t+1}(\mathrm{c})$ the noisy threshold foreground $F_{b i n}(\mathrm{~d})$

At the current step of algorithm we have 2 parameters $\alpha$ and $\beta$, which are necessary to be estimated. Optimum values of $\alpha$ and $\beta$ can be estimated using receiver operating characteristic 
(ROC) analysis. For estimation, implementation the training set from 5 video sequences of the 200 frames length which contain and do not contain smoke were used. Using the ground truth regions which have been online marked as a smoke in the training frames, rates of true and false detection were calculated for the whole frame set. We received a background for each value of $\alpha$ within a range of $(0,1)$. After that we applied a background subtraction and thresholding to each frame from a training set. And then we calculated True Positive Rate (TPR) and False Positive Rate (FPR) as follows:

$$
T P R=\frac{T P}{P} ; F P R=\frac{F P}{N},
$$

where TP - number of correctly classified pixels, $P$ - number of all positive classified pixels; $F P$ - number of incorrectly classified pixels, $N$ - number of all negative classified pixels.

For each value of $\alpha$, the average TPR and FPR is evaluated on a training frame set and used in the ROC curve (Figure 2.4 ).

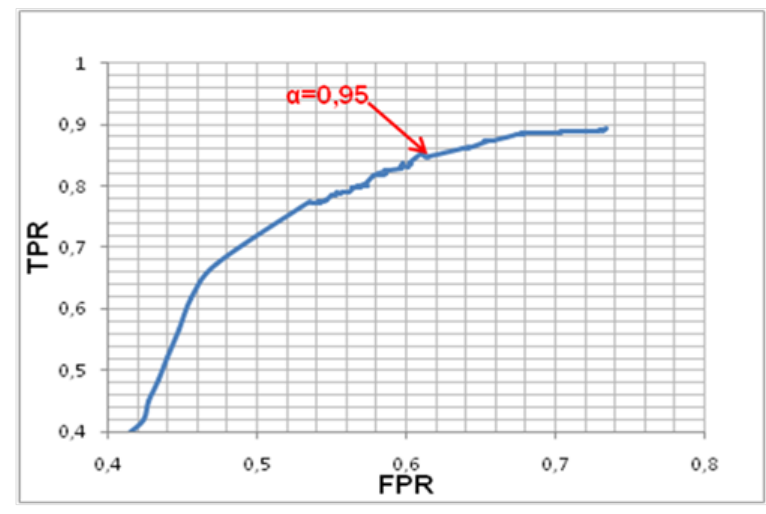

(a)

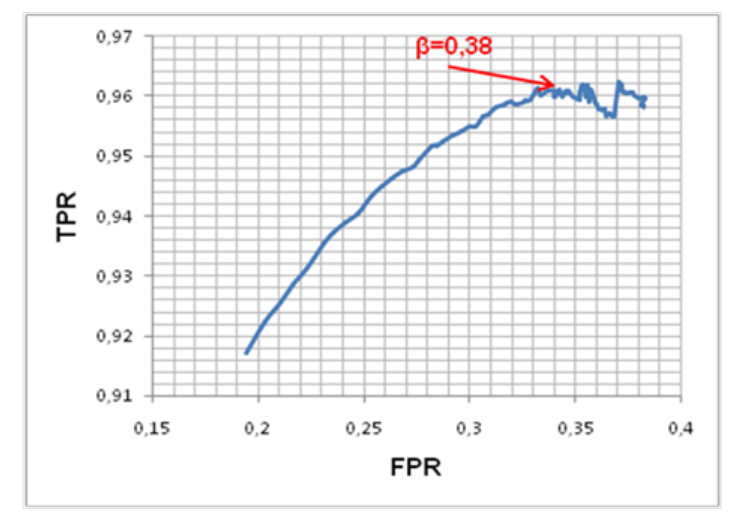

(b)

Figure 2.4. ROC curve for variable $\alpha$ (a) and $\beta$ (b)

Using the ROC curve, an optimum value for $\alpha$ can be easily selected for the smoke detection algorithm based on a predefined correct detection versus false detection rates. It is necessary to choose such value of $\alpha$ that slowly moving objects will not join a background too quickly, i.e. that a smoke will not fixed in a background too fast. At the given stage of algorithm high TPR is important and high enough $F P R$ is acceptable as it is necessary to receive as much as possible pixels for the analysis, and incorrectly classified pixels should be excluded at the following stages. Therefore, we have established an $\alpha$ value equals to 0.95. Similarly, using the training frame set, receiving a foreground component $F_{t+1}$ and after that the foreground $F_{b i n}$ and counting $F P R$ and $T P R$ for all values $\beta$ from a range $(0,1)$ with the step 0.001 , we build a ROC curve for $\beta$ (Figure 2.4 ). Value of $\beta$ has been chosen to be equal to 0.38 , because at such value of $\beta$ provides high TPR and low FPR.

\subsection{Connected component analysis}

On the next step of algorithm to clear of noise and to connect of moving blobs, the connected components analysis is used. This form of analysis takes in a noisy input foreground. Morphological operations are applied to reduce the noise: 
- morphological opening to shrink areas of small noise:

$$
S \circ M=(S(-) M) \oplus M,
$$

where $S$ - image, $M$ - structuring element $3 \times 3$;

- morphological closing to rebuild the area of surviving components that was lost in opening:

$$
S \bullet M=(S \oplus M)(-) M,
$$

where $M$ - structuring element $3 \times 3$.

Then search of all contours is carried out. It tosses the contours that are too small and approximate the rest with polygons. The figure 2.5a shows the results of connected components analysis.

\subsection{Moving blobs classification}

Blocks matching approach for optical flow calculation assumes that the frame is divided into small regions called blocks. It considers a primary direction of smoke propagation. In [16] it is shown that global direction of smoke is $0-45^{\circ}$. This statement allows to simplify procedure of blocks matching detection and, hence, considerably to reduce number of calculations. Blocks are typically squares and contain some number of pixels. These blocks do not overlap. In our implementation frames in the size $320 \times 240$ pixels divided into blocks $2 \times 2$ pixels. Block matching algorithm attempt to divide both the previous and current frames into such blocks and then compute the motion of these blocks. Each block of size $2 \times 2$ can move in eight possible directions (Figure 2.5p).

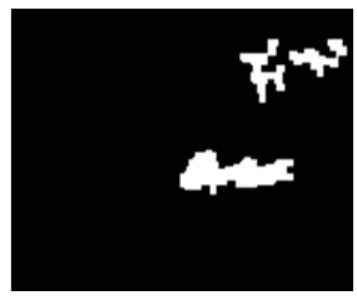

(a)

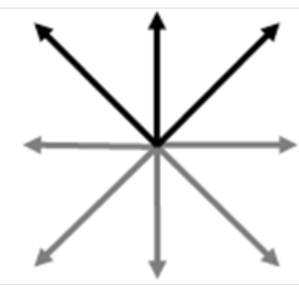

(b)

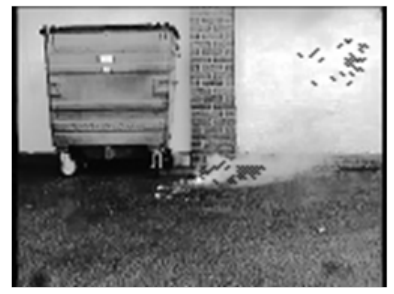

(c)

Figure 2.5. The clean up foreground (a) by the connected components analysis, the vectors having a primary direction of smoke propagation (b) and the results of optical flow calculation (c)

Our implementation searches in three directions (the black vectors on figure $2.5 \mathrm{~b}$ ) of the original block $q_{x, y}^{P R E V}$ (in the previous frame) and compares the candidate new blocks $q_{x-1, y-1}^{\text {curr }}, q_{x, y-1}^{\text {curr }}$ and $q_{x+1, y-1}^{\text {curr }}$ (in the current frame) with the original block. This comparison is calculated as follows:

$$
F\left(q_{x, y}^{P R E V}, q_{x+k, y-1}^{c u r r}\right)_{x, y \in[2 ; N]}^{k i n\{-1 ; 0 ; 1\}}=\left(\frac{\min \left(I_{i, j}^{P R E V}, I_{i, j}^{c u r r}\right)}{\max \left(I_{i, j}^{P R E V}, I_{i, j}^{c u r r}\right)}\right),
$$

where $I_{i, j}^{P R E V}$ is the intensity value of pixel on the previous frame, belonging to the block $q_{x, y}^{P R E V}, I_{i, j}^{c u r r}$ is the intensity value of pixel on the current frame, belonging to the block $q_{x, y}^{\text {curr }}$, 
$N$ is count of blocks into which divided the previous and current frame. The block $q_{x, y}^{P R E V}$ in the previous frame will correspond to the block in the current frame if function $F$ has the maximum value. Optical flow calculation (function $F$ ) is done only for the blocks belonging to the foreground (Figure 2.5p). The result of this step is the set of vectors having a direction corresponding to primary propagation of smoke (Figure 2.5.).

From each blob in the previous steps we calculate percentage $\rho$ of blocks, which have moved in primary direction of smoke:

$$
\rho=\frac{c_{s}}{c} \bullet 100 \%,
$$

where $c$ - the total number of blocks on a current frame, and Weber contrast $C_{w}$ :

$$
C_{w}=\frac{1}{n} \sum_{i=1}^{n} \frac{F_{t+1}(x, y)-B_{t}(x, y)}{B_{t}(x, y)},
$$

where $F_{t+1}(x, y)$ - value of pixel intensity $(x, y)$ at time instant $t$, belonging to a blob, $B_{t}(x, y)$ - value of background pixel intensity $(x, y)$ at time instant $t$ under blob, $n$ - number of the pixels belonging to a blob. If the blob has successfully checked out, then we classify it as a smoke. Experimentally established values $C_{w}>0,5$ and $\rho>20 \%$ allow efficient distinguishing a smoke from objects with similar behavior: a fog, shadows from slowly moving objects and patches of light.

\section{RESULTS AND DISCUSSION}

The developed algorithm was tested on the real cases. Test were run on a PC (Pentium(R) DualCore CPU T4300, 2,1 GHz, RAM 1,96GB). Our program was implemented using Visual $\mathrm{C}++$ and an open source computer vision library OpenCV. The proposed algorithm has been evaluated using data set publicly available at the web address http://signal.ee.bilkent.edu.tr /VisiFire/Demo/SampleClips.html and http://www.openvisor.org. Test video sequences contain a smoke, moving people, moving transport, a complex dynamic background, and also a number of video sequences without any smoke. Figure 3.6 shows some examples of smoke detection.

Detection results for some of the test sequences are presented in Table 1. Processing time of a current frame depends on the blob sizes and frequency of changes occurring in a background. If the background is stable and few blobs are detected then processing time decreases. Table 1 (the second column) contains average processing time on all frames for each test video sequence. The smoke has been found successfully out on all test video sequences with a smoke.

If at the moment of appearance on a scene a smoke moves slowly and is strongly rarefied (sequences b, d, h), then it is gradually included in the background. Therefore in this case, we cannot directly find out a smoke and detection time is increasing. The performed experiments have shown that the algorithm quickly finds out a smoke on a complex dynamic scene. Smoke detection is achieved practically in real time. The processing time per frame is about $15 \mathrm{~ms}$. for frames with sizes of 320 by 240pixels. The algorithm considers both dynamic and static features of a smoke.

The algorithm has a low false alarm level. False alarms on objects with properties similar to a smoke are sometimes possible. For example, with such properties as moving clouds (figure 3.6.). Tracing of smoky properties during some frames can solve this problem. 


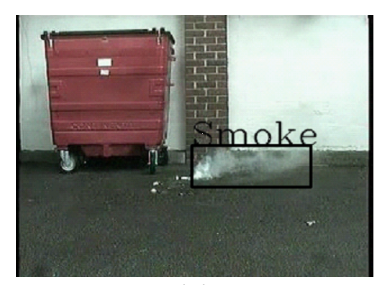

(a)

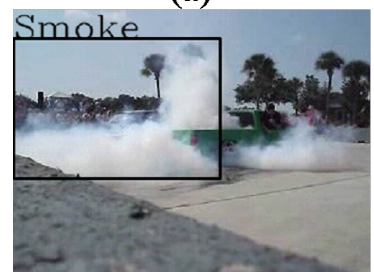

(e)

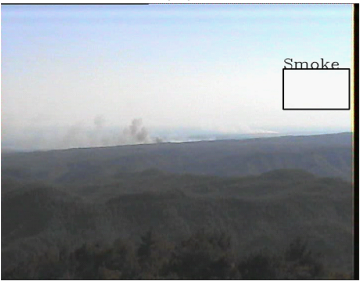

(j)

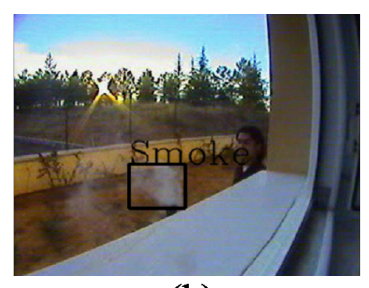

(b)

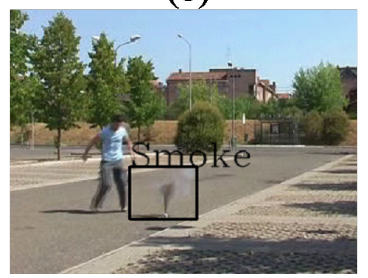

(f)

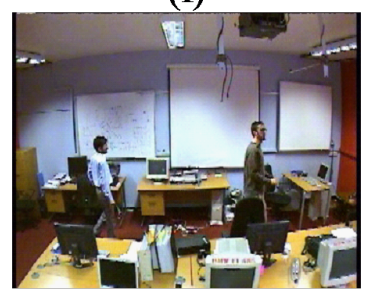

(k)

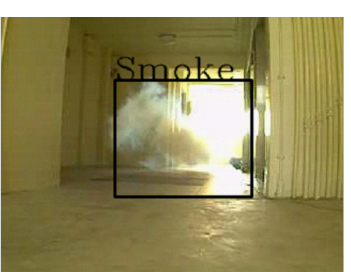

(c)

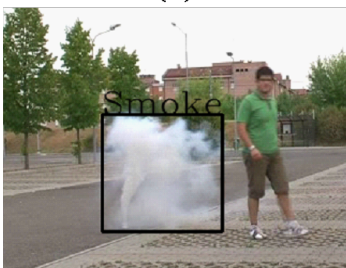

(g)

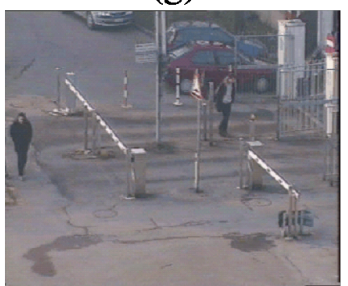

(1)

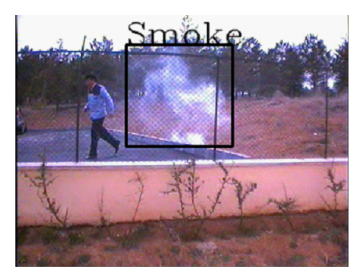

(d)

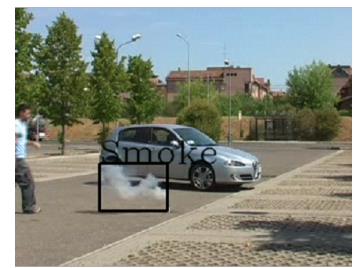

(h)

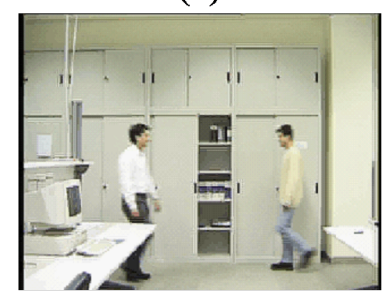

(m)

Figure 3.6. Smoke detection in real video sequences

Table 1. Detection results of our algorithm for some of the test sequences

\begin{tabular}{|c|c|c|c|c|}
\hline $\begin{array}{l}\text { Video } \\
\text { sequences } \\
\text { (Figure } 3.6\end{array}$ & $\begin{array}{c}\text { The } \\
\text { processing } \\
\text { time per } \\
\text { frame }(\mathrm{ms})\end{array}$ & $\begin{array}{l}\text { The smoke was } \\
\text { presented with/ } \\
\text { is found with } \\
\text { (number of frame) }\end{array}$ & $\begin{array}{c}\text { The count of } \\
\text { frames false alarm/ } \\
\text { on which was } \\
\text { total of frames }\end{array}$ & Description \\
\hline $\mathrm{a}$ & 12.665 & $10 / 12$ & $0 / 900$ & $\begin{array}{l}\text { Smoke from a smoke pot } \\
\text { against a white wall }\end{array}$ \\
\hline $\mathrm{b}$ & 14.786 & $20 / 112$ & $0 / 244$ & $\begin{array}{l}\text { Smoke in a waste basket } \\
\text { monitored from an indoor camera }\end{array}$ \\
\hline $\mathrm{c}$ & 14.969 & $80 / 87$ & $0 / 483$ & Smoke indoors \\
\hline $\mathrm{d}$ & 15.003 & $30 / 117$ & $0 / 630$ & Smoke in a garden \\
\hline $\mathrm{e}$ & 15.491 & $360 / 388$ & $0 / 2200$ & Smoke on parking lot \\
\hline $\mathrm{f}$ & 14.039 & $463 / 469$ & $0 / 1835$ & $\begin{array}{l}\text { Smoke from a smoke pot and } \\
\text { the walking man a sunny day }\end{array}$ \\
\hline $\mathrm{g}$ & 15.511 & $398 / 400$ & $0 / 2151$ & $\begin{array}{l}\text { Smoke from a smoke pot and } \\
\text { the walking man an overcast day }\end{array}$ \\
\hline $\mathrm{h}$ & 14.636 & $500 / 657$ & $0 / 1935$ & $\begin{array}{l}\text { Smoke from a smoke pot, the } \\
\text { walking man and the driving off car }\end{array}$ \\
\hline $\mathrm{j}$ & 13.546 & - & $5 / 600$ & Monitoring forest fires \\
\hline $\mathrm{k}$ & 16.346 & - & $0 / 1073$ & Two men walking in a room \\
\hline 1 & 14.567 & - & $0 / 1179$ & A parking lot and walking people \\
\hline $\mathrm{m}$ & 15.719 & - & $0 / 887$ & Two men walking in a light room \\
\hline
\end{tabular}


The smoke and flame are primary signs of a fire. Often there is a visible smoke development prior to flame. It can be important for early fire prevention. Therefore, our algorithm can be effectively used in video surveillance systems for early detection of fire on open spaces.

\section{CONCLUSION}

We have presented in this paper an algorithm for smoke detection in video sequences. Our algorithm consists of the following steps: preprocessing; slowly moving areas and pixels segmentation in a current input frame based on adaptive background subtraction; merge slowly moving areas with pixels into blobs; classification of the blobs obtained before. We use adaptive background subtraction at a stage of moving detection. Moving blobs classification is based on optical flow calculation, Weber contrast analysis and takes into account primary direction of smoke propagation. The efficiency of our approach is illustrated and confirmed by experimental videos.

\section{REFERENCES}

[1] D. Han, B. Lee, Flame and smoke detection method for early real-time detection of a tunnel fire, Fire Safety Journal 44 (2009) 951-961.

[2] W. Phillips III, M. Shah, N. V. Lobo, Flame recognition in video, Pattern Recognition Letters 23 (2002) 319-327.

[3] Y. G. Sahin, T. Ince, Early Forest Fire Detection Using Radio-Acoustic Sounding System, Sensors 9 (2009) 1485-1498.

[4] A. M. Fernandes, A. B. Utkin, A. V. Lavrov, R. M. Vilar, Design of committee machines for classification of single-wavelength lidar signals applied to early forest fire detection, Pattern Recognition Letters 26 (2005) 625-632.

[5] B. U. Toreyin, Y. Dedeoglu, U. Gudukbay, Computer vision based method for real-time fire and flame detection, Pattern recognition letters (2006) 49-58.

[6] P. Piccinini, S. Calderara, R. Cucchiara, Reliable smoke detection system in the domains of image energy and color, 15th International Conference on Image Processing (2008) 1376-1379.

[7] B. Toreyin, et al, Wavelet based real-time smoke detection in video, Signal Processing: Image communication, EURASIP, Elsevier.20 (2005) 55-256.

[8] D. Kim, Y. F. Wang, Smoke Detection in Video, World Congress on Computer Science and Information Engineering (2009) 759-763.

[9] F. Comez-Rodriuez, et al, Smoke Monitoring and measurement Using Image Processing. Application to Forest Fires. In:Automatic Target Recognation XIII, Proceedings of SPIE 5094, 2003 (404-411).

[10] D. Kristini, T. Jakovevi, D. Stipaniev, Histogram-Based Smoke Segmentation in Forest Fire Detection System, Information Technology and Control 38 (3) (2009) 237-244. 
[11] F. Yuan., A fast accumulative motion orientation model based on integral image for video smoke detection, Pattern Recognition Letters 29 (7)(2008) 925-932.

[12] T. Amiaz, S. Fazekas, D. Chetverikov, N. Kiryati, Detecting regions of dynamic texture, First International Conference on Scale Space and Variational Methods in Computer Vision, 2007 (848-859).

[13] T. Crivelli, B. Cernuschi-Frias, P. Bouthemy, J. Yao, Mixed-state causal modeling for statistical KL-based motion texture tracking, Pattern Recognition Letters 3114 (2010) 2286-2294.

[14] E. Stolnitz, T. DeRose, D. Salesin, Wavelets for Computer Graphics: Theory and Applications, Morgan Kaufmann.

[15] R. T. Collins, A system for video surveillance and monitoring, Proc. of American Nuclear Society 8th Int. Topical Meeting on Robotics and Remote Systems, 68-73.

[16] Rubaiyat Yasmin, Detection of Smoke Propagation Direction Using Color Video Sequences, 2009.

[17] T. Porter, T. Duff, Compositing digital images, Computer Graphics 183 (1984) 253-259.

Received on May 22, 2012 Revised on December 22, 2012 